\title{
On Transitive Points in a Generalized Shift Dynamical System
}

\author{
Bahman Taherkhani ${ }^{1}$ and Fatemah Ayatollah Zadeh Shirazi ${ }^{2}$ \\ ${ }^{1}$ Department of Mathematics, Tarbiat Modares University, P.O. Box 14115-137, Tehran, Iran \\ ${ }^{2}$ Faculty of Mathematics, Statistics and Computer Science, College of Science, University of Tehran, \\ Enghelab Avenue, Tehran, Iran \\ Correspondence should be addressed to Bahman Taherkhani; bahman.taherkhani@modares.ac.ir
}

Received 31 August 2014; Accepted 1 December 2014

Academic Editor: Yonghui Xia

Copyright (C) 2015 B. Taherkhani and F. Ayatollah Zadeh Shirazi. This is an open access article distributed under the Creative Commons Attribution License, which permits unrestricted use, distribution, and reproduction in any medium, provided the original work is properly cited.

Considering point transitive generalized shift dynamical system $\left(X^{\Gamma}, \sigma_{\varphi}\right)$ for discrete $X$ with at least two elements and infinite $\Gamma$, we prove that $X$ is countable and $\Gamma$ has at most $2^{\aleph_{0}}$ elements. Then, we find a transitive point of the dynamical system $\left(\mathbb{N}^{\mathbb{N} \times \mathbb{Z}}, \sigma_{\tau}\right)$ for $\tau: \mathbb{N} \times \mathbb{Z} \rightarrow \mathbb{N} \times \mathbb{Z}$ with $\tau(n, m)=(n, m+1)$ and show that point transitive $\left(X^{\Gamma}, \sigma_{\varphi}\right)$, for infinite countable $\Gamma$, is a factor of $\left(\mathbb{N}^{\mathbb{N} \times \mathbb{Z}}, \sigma_{\tau}\right)$.

\section{Introduction}

By a topological dynamical system we mean $(Z, h)$ where $Z$ is a topological space and $h: Z \rightarrow Z$ is a continuous map. In the dynamical system $(Z, h)$ for $z_{0} \in Z$, we call $\left\{h^{i}\left(z_{0}\right): i \geq 0\right\}$ the orbit of $z_{0} \in Z$ and denote it by $O_{h}\left(z_{0}\right)$. Also we call the dynamical system $(Z, h)$ point transitive if there exists $z_{0} \in Z$ with $\overline{O_{h}\left(z_{0}\right)}=Z$; moreover, we call $z_{0}$ a transitive point. Moreover, we call $(Z, h)$ topologically transitive if for every nonempty open subset $U, V$ of $Z$ there exists $n \geq 1$ with $h^{n}(U) \cap V \neq \varnothing$.

For arbitrary map $\varphi: \Gamma \rightarrow \Gamma$ and nonvoid sets $X, \Gamma$, define $\sigma_{\varphi}: X^{\Gamma} \rightarrow X^{\Gamma}$ by $\sigma_{\varphi}\left(\left(x_{\gamma}\right)_{\gamma \in \Gamma}\right)=\left(x_{\varphi(\gamma)}\right)_{\gamma \in \Gamma}$ for all $\left(x_{\gamma}\right)_{\gamma \in \Gamma} \in X^{\Gamma}$. If $X$ is a topological space and $X^{\Gamma}$ is equipped with the product (pointwise convergence) topology, then $\sigma_{\varphi}$ : $X^{\Gamma} \rightarrow X^{\Gamma}$ is continuous; we call $\left(X^{\Gamma}, \sigma_{\varphi}\right)$ a generalized shift dynamical system. Generalized shifts in this sense have been introduced for the first time in [1]. So many dynamical and nondynamical properties of generalized shifts like algebraic entropy, topological entropy, and Devaney chaos [2] of a generalized shift have been studied recently.

One of the famous dynamical systems is the one-sided shift dynamical system $\left(\{1, \ldots, k\}^{\mathbb{N}}, \sigma\right)$ where $k \geq 2$ and $\sigma\left(\left(x_{n}\right)_{n \in \mathbb{N}}\right)=\left(x_{n+1}\right)_{n \in \mathbb{N}}$ for all $\left(x_{n}\right)_{n \in \mathbb{N}} \in\{1, \ldots, k\}^{\mathbb{N}}$; we recall once more the one-sided shift in our final example.

It is well known that, in dynamical system $(Z, h)$ with Hausdorff $Z$, if $Z$ does not have any isolated point and it is point transitive, then $(Z, h)$ is topological transitive [3, Proposition 1]; however, as it has been mentioned in [3], two concepts, topological transitivity and point transitivity, are not equivalent in general (one may find notes regarding these two concepts (and their historical points of view) in [4]; however, the reader will find $[5,6]$ interesting too). Moreover, for discrete $X$ with at least two elements, infinite countable $\Gamma$, and $\varphi: \Gamma \rightarrow \Gamma$, if the generalized shift dynamical system $\left(X^{\Gamma}, \sigma_{\varphi}\right)$ is topological transitive, then $\varphi: \Gamma \rightarrow \Gamma$ is one to one without periodic points [2, Theorem 2.11]; the main aim of the following text is to construct a transitive point of $\left(X^{\Gamma}, \sigma_{\varphi}\right)$ in the above case. We recall that $\alpha \in \Gamma$ is a periodic point of $\varphi: \Gamma \rightarrow \Gamma$ if there exists $n \geq 1$ such that $\varphi^{n}(\alpha)=\alpha$.

In the following text, suppose $X$ is discrete with at least two elements, $\Gamma$ is an infinite set, and $\varphi: \Gamma \rightarrow \Gamma$ is an arbitrary map. Also suppose $\mathbb{N}=\{1,2, \ldots\}$ is the set of all natural numbers, $\mathbb{Z}=\{0, \pm 1, \pm 2, \ldots\}$ is the set of all integers, and $\aleph_{0}=\operatorname{card}(\mathbb{N})$, and we use the symbol $c$ for $2^{\aleph_{0}}$. 


\section{Two Cardinal Bounds in the Point Transitive Generalized Shift Dynamical Systems}

In this section, we prove that, for discrete $X$ with at least two elements, infinite $\Gamma$, and arbitrary map $\varphi: \Gamma \rightarrow \Gamma$, if the generalized shift dynamical system $\left(X^{\Gamma}, \sigma_{\varphi}\right)$ is point transitive, then

(i) the map $\varphi: \Gamma \rightarrow \Gamma$ is one to one without periodic point;

(ii) the set $X$ is countable, and $\operatorname{card}(\Gamma) \leq c$.

Theorem 1. If $\left(X^{\Gamma}, \sigma_{\varphi}\right)$ is point transitive, then $X$ is countable.

Proof. Assume that $X$ is uncountable, and let $b=\left(b_{\alpha}\right)_{\alpha \in \Gamma} \in$ $X^{\Gamma}$. Choose $\beta \in \Gamma$ and let $Y=\left\{b_{\varphi^{n}(\beta)}: n \geq 0\right\}$. There exists $c \in X \backslash Y$ since $X$ is uncountable and $Y$ is countable. For $V=\left\{\left(x_{\alpha}\right)_{\alpha \in \Gamma}: x_{\beta}=c\right\}, V$ is an open subset of $X^{\Gamma}$ such that

$$
V \cap\left\{\sigma_{\varphi}^{n}\left(\left(b_{\alpha}\right)_{\alpha \in \Gamma}\right): n \geq 0\right\}=\varnothing
$$

therefore, $V \cap O_{\sigma_{\varphi}}(b)=\varnothing$ and $X^{\Gamma} \neq \overline{O_{\sigma_{\varphi}}(b)}$; hence, $\left(X^{\Gamma}, \sigma_{\varphi}\right)$ is not point transitive.

Definition 2. Define $\sim_{\varphi}$ relation on $\Gamma$ letting

$$
\alpha \sim{ }_{\varphi} \beta \Longleftrightarrow\left(\exists n, m \in \mathbb{N}\left(\varphi^{n}(\alpha)=\varphi^{m}(\beta)\right)\right)
$$

for $\alpha, \beta \in \Gamma$. It is easy to verify that $\sim_{\varphi}$ is an equivalence relation on $\Gamma$.

As a matter of fact, for injective $\varphi: \Gamma \rightarrow \Gamma$ and $\alpha \in \Gamma$, we have $\alpha / \sim_{\varphi}=\bigcup\left\{\varphi^{n}(\alpha): n \in \mathbb{Z}\right\}$ (where $\alpha / \sim_{\varphi}=\{\beta \in \Gamma$ : $\left.\left.\alpha \sim{ }_{\varphi} \beta\right\}\right)$ so exactly one of the following cases occurs:

(I) there exists $n \geq 1$ with $\varphi^{n}(\alpha)=\alpha$ ( $\alpha$ is a periodic point of $\varphi$ ),

(II) the sequence $\left\{\varphi^{n}(\alpha)\right\}_{n \geq 1}$ is one to one and there exists $k \geq 1$ with $\varphi^{-k}(\alpha)=\varnothing$,

(III) the sequence $\left\{\varphi^{n}(\alpha)\right\}_{n \geq 1}$ is one to one and for all $k \geq 1$ we have $\varphi^{-k}(\alpha) \neq \varnothing$.

Now one can verify that the above cases are equivalent to the following statements in the corresponding case:

(I) $\alpha / \sim_{\varphi}$ is finite (and equal to $\left\{\varphi^{i}(\alpha): i \geq 0\right\}$ ),

(II) $\alpha / \sim_{\varphi}$ is infinite and there exists $\beta \in \Gamma$ with $\alpha / \sim_{\varphi}=$ $\left\{\varphi^{i}(\beta): i \geq 0\right\}$,

(III) $\alpha / \sim_{\varphi}$ is infinite and there exists a (one to one) bisequence $\left\{b_{n}\right\}_{n \in \mathbb{Z}}$ such that $\alpha / \sim_{\varphi}=\left\{b_{n}: n \in \mathbb{Z}\right\}$ and for all $n \in \mathbb{Z}$ we have $b_{n+1}=\varphi\left(b_{n}\right)$.

In particular, in all of the above cases, $\alpha / \sim_{\varphi}$ is countable.

Theorem 3. For $\varphi: \Gamma \rightarrow \Gamma$, if $\left(X^{\Gamma}, \sigma_{\varphi}\right)$ is point transitive, then $\operatorname{card}(\Gamma) \leq c$.
Proof. Let $\left(X^{\Gamma}, \sigma_{\varphi}\right)$ be point transitive; then, as it has been mentioned in the Introduction, by [2, Theorem 2.11], $\varphi$ : $\Gamma \rightarrow \Gamma$ is one to one without periodic points. Now suppose $w=\left(x_{\alpha}\right)_{\alpha \in \Gamma} \in X^{\Gamma}$ is a transitive point of $X^{\Gamma}$. Using Theorem 1 , $X$ is countable; also $\varphi: \Gamma \rightarrow \Gamma$ is one to one without periodic point. Consider $\sim_{\varphi}$ as in Definition 2 . Let $\Lambda:=\Gamma / \sim_{\varphi}$; using the Axiom of Choice there exists a choice function $\omega: \Lambda \rightarrow$ $\bigcup \Lambda=\Gamma$; thus, for all $D \in \Lambda$, we have $\omega(D) \in D$; we denote $\omega(D)$ simply by $\alpha_{D}$. Since

$$
\overline{O_{\sigma_{\varphi}}(w)}=\overline{\left\{\left(x_{\varphi^{n}(\alpha)}\right)_{\alpha \in \Gamma}: n \geq 0\right\}}=X^{\Gamma}
$$

for distinct $D, G \in \Lambda$ we have $\left(x_{\varphi^{n}\left(\alpha_{D}\right)}\right)_{n \in \mathbb{N}} \neq\left(x_{\varphi^{n}\left(\alpha_{G}\right)}\right)_{n \in \mathbb{N}}$. Define $\xi: \Lambda \rightarrow X^{\mathbb{N}}$ with $\xi(D)=\left(x_{\varphi^{n}\left(\alpha_{D}\right)}\right)_{n \in \mathbb{N}}($ for $D \in \Lambda)$. The map $\xi: \Lambda \rightarrow X^{\mathbb{N}}$ is one to one; otherwise, there exist distinct $D, G \in \Lambda$ with $\xi(D)=\xi(G)$, so for all $n \geq 1$ we have $x_{\varphi^{n}\left(\alpha_{D}\right)}=x_{\varphi^{n}\left(\alpha_{G}\right)}$ and

$$
\begin{aligned}
\overline{O_{\sigma_{\varphi}}(w)} & =\overline{\left\{\sigma_{\varphi}^{n}\left(\left(x_{\alpha}\right)_{\alpha \in \Gamma}\right): n \geq 0\right\}} \\
& =\overline{\left\{\left(x_{\varphi^{n}(\alpha)}\right)_{\alpha \in \Gamma}: n \geq 0\right\}} \\
& \subseteq \overline{\left\{\left(y_{\alpha}\right)_{\alpha \in \Gamma} \in X^{\Gamma}: y_{\alpha_{D}}=y_{\alpha_{G}}\right\}} \\
& =\left\{\left(y_{\alpha}\right)_{\alpha \in \Gamma} \in X^{\Gamma}: y_{\alpha_{D}}=y_{\alpha_{G}}\right\} \neq X^{\Gamma}
\end{aligned}
$$

which is a contradiction. Therefore, $\xi: \Lambda \rightarrow X^{\mathbb{N}}$ is one to one, and (note the fact that every $D \in \Lambda$ is countable, by injection of $\varphi: \Gamma \rightarrow \Gamma$ )

$$
\begin{aligned}
\operatorname{card}(\Gamma) & =\operatorname{card}(\bigcup \Lambda) \leq \aleph_{0} \operatorname{card}(\Lambda) \leq \aleph_{0} \operatorname{card}\left(X^{\mathbb{N}}\right) \\
& \leq \aleph_{0}\left(\aleph_{0}^{\aleph_{0}}\right)=2^{\aleph_{0}}=c .
\end{aligned}
$$

\section{Towards the Main Construction}

In the first part of this section, we construct a point transitive generalized shift dynamical system $\left(\mathbb{N}^{\mathbb{N} \times \mathbb{Z}}, \sigma_{\tau}\right)$ which plays a key role in the final part and our main construction.

3.1. Construction of a Point Transitive Generalized Shift Dynamical System. We denote the collection of $n \times m$ matrices with the arrays in an arbitrary set $A$, by $\operatorname{Mat}_{n \times m}(A)$.

Definition 4. For set $A=\left\{x_{1}, \ldots, x_{n}\right\}$ with $n$ elements, suppose

$$
M(A) \in \operatorname{Mat}_{n \times n^{n}}(A)
$$

is the following matrix up to rearrangement of $x_{1}, \ldots, x_{n}$ : 


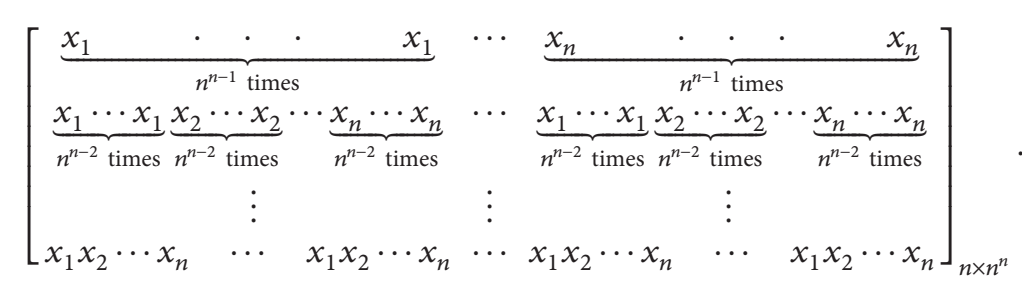

However, $M(A)$ is well defined up to the rearrangement of $x_{1}, \ldots, x_{n}$; that is, if $\varrho:\{1, \ldots, n\} \rightarrow\{1, \ldots, n\}$ is a permutation (on $\{1, \ldots, n\}$ ), one may consider the following matrix as $M(A)$ too, where $y_{i}=x_{\rho(i)}$ for $i=1, \ldots, n$ (see Example 5):

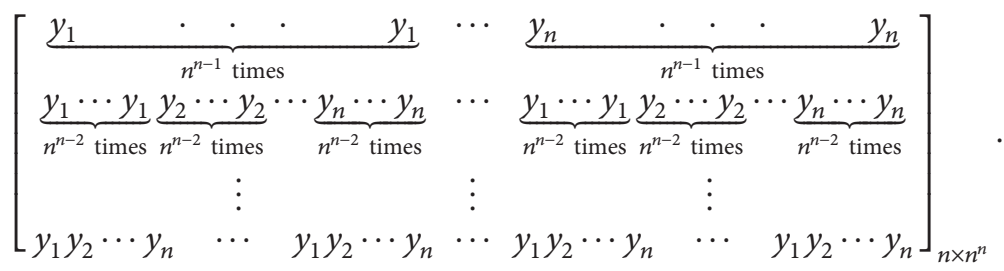

Also, note that the columns of $M(A)$ are pairwise distinct.

Example 5. For $A=\{1,2\}, M(A)$ is any of the following matrices:

$$
\left[\begin{array}{llll}
1 & 1 & 2 & 2 \\
1 & 2 & 1 & 2
\end{array}\right], \quad\left[\begin{array}{llll}
2 & 2 & 1 & 1 \\
2 & 1 & 2 & 1
\end{array}\right] .
$$

Note 1. Under the same notations as in Definition 4, if $M(A)=\left[a_{(i, j)}\right]_{n \times n^{n}}$ and $b_{1}, \ldots, b_{n} \in A$, then there exists (a unique) $j \in\left\{1, \ldots, n^{n}\right\}$ such that

$$
\left[\begin{array}{c}
a_{1 j} \\
a_{2 j} \\
\vdots \\
a_{n j}
\end{array}\right]=\left[\begin{array}{c}
b_{1} \\
b_{2} \\
\vdots \\
b_{n}
\end{array}\right] .
$$

Definition 6. For $p \in \mathbb{N}, A:=\{1, \ldots, p\}$ and $B:=A^{p}$; then, $M(B) \in \operatorname{Mat}_{\left(p^{p}\right) \times\left(p^{p}\right)^{\left(p^{p}\right)}}(B)$; in other words, $M(B)$ is a matrix whose arrays belong to $\operatorname{Mat}_{1 \times p}(A)$. By removing partitions of the matrix $M(B)$, we have a new matrix $K_{p} \in$ Mat $_{\left(p^{p}\right) \times p\left(p^{p}\right)^{\left(p^{p)}\right.}}(A)$.

For example, let $p=2$; then, $A=\{1,2\}$ and $B=$ $\{(1,1),(1,2),(2,1),(2,2)\}$ and we have the following matrix as $M(B)$ :

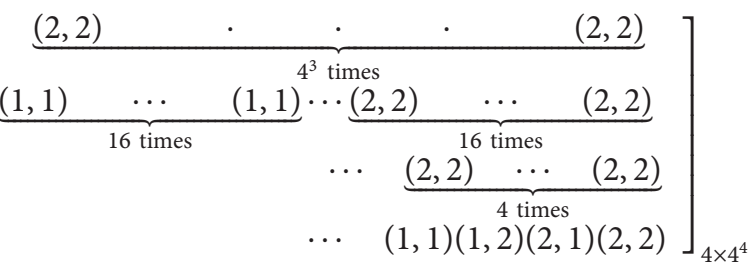

so $K_{2}$ is the following matrix:
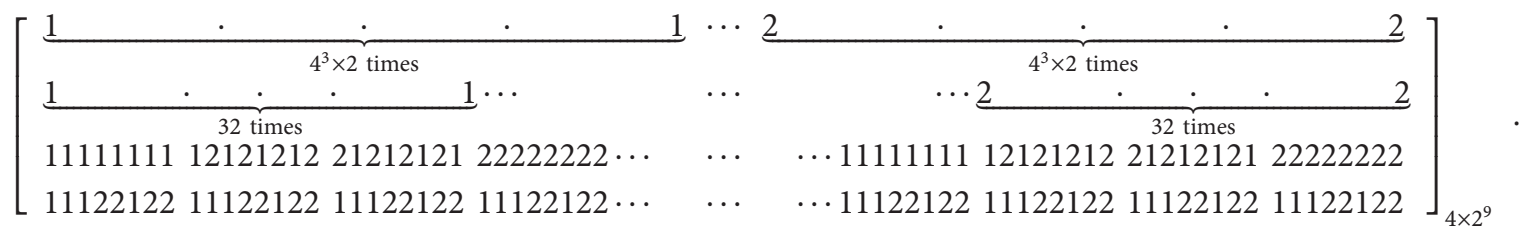
Note 2. Using Note 1 and Definition 6 , if $\Delta=\left[d_{(i, j)}\right]_{n \times n}$ is an $n \times n$ matrix with arrays in $\{1, \ldots, n\}$ and $K_{n}=$ $\left[k_{i j}^{n}\right]_{n^{n} \times n\left(n^{n}\right)^{\left(n^{n}\right)}}$ (introduced in Definition 6), then there exists $t \in\left\{0,1, \ldots, n\left(n^{n}\right)^{\left(n^{n}\right)}-1\right\}$ such that

$$
\left[\begin{array}{cccc}
k_{1(t+1)}^{n} & k_{1(t+2)}^{n} & \cdots & k_{1(t+n)}^{n} \\
k_{2(t+1)}^{n} & k_{2(t+2)}^{n} & \cdots & k_{2(t+n)}^{n} \\
\vdots & \vdots & & \vdots \\
k_{n(t+1)}^{n} & k_{n(t+2)}^{n} & \cdots & k_{n(t+n)}^{n}
\end{array}\right]=\left[\begin{array}{cccc}
d_{(1,1)} & d_{(1,2)} & \cdots & d_{(1, n)} \\
d_{(2,1)} & d_{(2,2)} & \cdots & d_{(2, n)} \\
\vdots & \vdots & & \vdots \\
d_{(n, 1)} & d_{(n, 2)} & \cdots & d_{(n, n)}
\end{array}\right] .
$$

However there exists a unique column in $M\left(\{1, \ldots, n\}^{n}\right)$ equal to

$$
\begin{array}{r}
\left(\begin{array}{llll}
d_{(1,1)} & d_{(1,2)} & \cdots & d_{(1, n)}
\end{array}\right) \\
\left(\begin{array}{lllll}
d_{(2,1)} & d_{(2,2)} & \cdots & d_{(2, n)}
\end{array}\right) \\
\left(\begin{array}{llll}
d_{(n, 1)} & d_{(n, 2)} & \cdots & d_{(n, n)}
\end{array}\right) \\
n^{n}-n \text { times }\left\{\begin{array}{cccc}
(1 & 1 & \cdots & 1
\end{array}\right) \\
\\
\end{array}
$$

Lemma 7. If $V$ is a nonempty open subset of $\mathbb{N}^{\mathbb{N} \times \mathbb{Z}}$, then there exist $N \in \mathbb{N}$ and $\Delta=\left[d_{(i, j)}\right]_{1 \leq i \leq 2 N+1,-N \leq j \leq N}$ with $1 \leq d_{(i, j)} \leq$ $N$ for all $i, j$, such that

$$
\begin{aligned}
& \left\{\left(x_{(i, j)}\right)_{(i, j) \in \mathbb{N} \times \mathbb{Z}} \in \mathbb{N}^{\mathbb{N} \times \mathbb{Z}}:\right. \\
& \left.\forall i \in\{1, \ldots, 2 N+1\} \forall j \in\{-N, \ldots, N-1, N\} x_{(i, j)}=d_{(i, j)}\right\}
\end{aligned}
$$

is a subset of $V$.

Proof. Suppose $V$ is a nonempty open subset of $\mathbb{N}^{\mathbb{N} \times \mathbb{Z}}$; here, we search for a suitable open subset of $V$, namely, $W$. Choose $\left(a_{(i, j)}\right)_{(i, j) \in \mathbb{N} \times \mathbb{Z}} \in V$. There exist $\left(i_{1}, j_{1}\right), \ldots,\left(i_{p}, j_{p}\right) \in \mathbb{N} \times \mathbb{Z}$ such that

$$
\begin{aligned}
V_{1}:=\left\{\left(x_{(i, j)}\right)_{(i, j) \in \mathbb{N} \times \mathbb{Z}} \in \mathbb{N}^{\mathbb{N} \times \mathbb{Z}}:\right. \\
\left.\forall k \in\{1, \ldots, p\} \quad x_{\left(i_{k}, j_{k}\right)}=a_{\left(i_{k}, j_{k}\right)}\right\} \subseteq V .
\end{aligned}
$$

Let

$$
\begin{gathered}
N=\max \left(\left\{i_{k}: k=1, \ldots, p\right\} \cup\left\{\left|j_{k}\right|: k=1, \ldots, p\right\}\right. \\
\left.\cup\left\{a_{\left(i_{k}, j_{k}\right)}: k=1, \ldots, p\right\}\right) \\
d_{(i, j)}= \begin{cases}a_{(i, j)} & \exists k \in\{1, \ldots, p\}(i, j)=\left(i_{k}, j_{k}\right) \\
1 & \text { otherwise }\end{cases} \\
W=\left\{\left(x_{(i, j)}\right)_{(i, j) \in \mathbb{N} \times \mathbb{Z}} \in \mathbb{N}^{\mathbb{N} \times \mathbb{Z}}:\right.
\end{gathered}
$$$$
\left.\forall i \in\{1, \ldots, 2 N+1\} \forall j \in\{-N, \ldots, N\} x_{(i, j)}=d_{(i, j)}\right\} .
$$

Since for all $k \in\{1, \ldots, p\}$ we have $i_{k} \leq N<2 N+1,\left|j_{k}\right| \leq$ $N$, and $a_{\left(i_{k}, j_{k}\right)}=d_{\left(i_{k}, j_{k}\right)}$, we have $W \subseteq V_{1}(\subseteq V)$. Moreover, using the definition of $N$ and $d_{(i, j)}$ s, we have $\max \left\{d_{(i, j)}: i \in\right.$ $\mathbb{N}, j \in \mathbb{Z}\} \leq N$.

Convention 1. Consider the matrix

$$
\Psi=\left(\psi_{(i, j)}\right)_{(i, j) \in \mathbb{N} \times \mathbb{N}}=\left[\begin{array}{ccc}
\psi_{(1,1)} & \psi_{(1,2)} & \cdots \\
\psi_{(2,1)} & \psi_{(2,2)} & \cdots \\
\psi_{(3,1)} & \psi_{(3,2)} & \cdots \\
\vdots & \vdots & \vdots
\end{array}\right]
$$

such that

$$
\Psi=\left[\begin{array}{ccccc}
K_{1} & K_{2} & K_{3} & K_{4} & \cdots \\
1 & & & & \\
& 1 & & & \\
& & 1 & & \\
\vdots & \vdots & \vdots & \vdots & \ddots \\
1 & 1 & 1 & 1 & \\
\vdots & \vdots & \vdots & \vdots &
\end{array}\right] \text {, }
$$

where $K_{n}$ is introduced in Definition 6. In other words, for $n \in \mathbb{N}$, if $K_{n}=\left[k_{i j}^{n}\right]_{n^{n} \times n\left(n^{n}\right)^{\left(n^{n}\right)}}$ (as in Definition 6), then

$$
\psi_{(i, j)}= \begin{cases}k_{i s}^{n} & 1 \leq i \leq n^{n}, j=s+\sum_{1 \leq p \leq(n-1)} p\left(p^{p}\right)^{\left(p^{p}\right)}, \\ & \text { otherwise. }\end{cases}
$$

Lemma 8. Let

$$
a_{(i, j)}= \begin{cases}\psi_{(i, j)} & i \geq 1, \quad j \geq 1 \\ 1 & i \geq 1, \quad j \leq 0\end{cases}
$$

Then, $\left(a_{(i, j)}\right)_{(i, j) \in \mathbb{N} \times \mathbb{Z}}$ is a transitive point of the generalized shift dynamical system $\left(\mathbb{N}^{\mathbb{N} \times \mathbb{Z}}, \sigma_{\tau}\right)$ for $\tau: \mathbb{N} \times \mathbb{Z} \rightarrow \mathbb{N} \times \mathbb{Z}$ with $\tau(n, m)=(n, m+1)$. In particular, $\left(\mathbb{N}^{\mathbb{N} \times \mathbb{Z}}, \sigma_{\tau}\right)$ is point transitive.

Proof. We just should prove that for all nonempty open subsets $V$ of $\mathbb{N}^{\mathbb{N} \times \mathbb{Z}}$ there exists $n \geq 0$ with $\sigma_{\tau}^{n}\left(\left(a_{(i, j)}\right)_{(i, j) \in \mathbb{N} \times \mathbb{Z}}\right)=$ $\left(a_{(i, j+n)}\right)_{(i, j) \in \mathbb{N} \times \mathbb{Z}} \in V$. Suppose $V$ is a nonempty open subset of $\mathbb{N}^{\mathbb{N} \times \mathbb{Z}}$. By Lemma 7 , there exist $N \in \mathbb{N}$ and $\Delta=$ $\left[d_{(i, j)}\right]_{0 \leq i \leq 2 N+1,-N \leq j \leq N}$ such that

$$
\left\{\left(x_{(i, j)}\right)_{(i, j) \in \mathbb{N} \times \mathbb{Z}} \in \mathbb{N}^{\mathbb{N} \times \mathbb{Z}}:\right.
$$

$\left.\forall i \in\{1, \ldots, 2 N+1\} \forall j \in\{-N, \ldots, N-1, N\} x_{(i, j)}=d_{(i, j)}\right\}$ 
is a subset of $V$, and $\max \left\{d_{(i, j)}: 1 \leq i \leq 2 N+1,-N \leq\right.$ $j \leq N\} \leq N<2 N+1$. Let $M:=(2 N+1)((2 N+$ $\left.1)^{(2 N+1)}\right)^{\left((2 N+1)^{(2 N+1)}\right)}-1$ and let $L:=\sum_{1 \leq p \leq 2 N} p\left(p^{p}\right)^{\left(p^{p}\right)}$. Using Note 2 for

$$
Q:=\left[\begin{array}{cccc}
d_{(1,-N)} & d_{(1,-N+1)} & \cdots & d_{(1, N)} \\
d_{(2,-N)} & d_{(2,-N+1)} & \cdots & d_{(2, N)} \\
\vdots & \vdots & & \vdots \\
d_{(2 N+1,-N)} & d_{(2 N+1,-N+1)} & \cdots & d_{(2 N+1, N)}
\end{array}\right],
$$

there exists $t \in\{0, \ldots, M\}$ such that

$$
Q=\left[\begin{array}{cccc}
k_{1(t+1)}^{2 N+1} & k_{1(t+2)}^{2 N+1} & \cdots & k_{1(t+2 N+1)}^{2 N+1} \\
k_{2(t+1)}^{2 N+1} & k_{2(t+2)}^{2 N+1} & \cdots & k_{2(t+2 N+1)}^{2 N+1} \\
\vdots & \vdots & & \vdots \\
k_{(2 N+1)(t+1)}^{2 N+1} & k_{(2 N+1)(t+2)}^{2 N+1} & \cdots & k_{(2 N+1)(t+2 N+1)}^{2 N+1}
\end{array}\right] .
$$

Now according to Convention 1, we have

$$
Q=\left[\begin{array}{cccc}
\psi_{(1, t+1+L)} & \psi_{(1, t+2+L)} & \cdots & \psi_{(1, t+2 N+1+L)} \\
\psi_{(2, t+1+L)} & \psi_{(2, t+2+L)} & \cdots & \psi_{(2, t+2 N+1+L)} \\
\vdots & \vdots & & \vdots \\
\psi_{(2 N+1, t+1+L)} & \psi_{(2 N+1, t+2+L)} & \cdots & \psi_{(2 N+1, t+2 N+1+L)}
\end{array}\right]
$$

which leads to

$$
Q=\left[\begin{array}{cccc}
a_{(1, t+1+L)} & a_{(1, t+2+L)} & \cdots & a_{(1, t+2 N+1+L)} \\
a_{(2, t+1+L)} & a_{(2, t+2+L)} & \cdots & a_{(2, t+2 N+1+L)} \\
\vdots & \vdots & & \vdots \\
a_{(2 N+1, t+1+L)} & a_{(2 N+1, t+2+L)} & \cdots & a_{(2 N+1, t+2 N+1+L)}
\end{array}\right]
$$

since $0 \leq t \leq M$ and using the definition of $a_{(i, j)}$ s.

So $a_{(p, q+N+t+L+1)}=d_{(p, q)}$ for $p \leq 2 N+1$ and $|q| \leq N$. Therefore, for $p \leq 2 N+1$ and $|q| \leq N$, we have

$$
\sigma_{\tau}^{N+t+L+1}\left(a_{(i, j)}\right)_{(i, j) \in \mathbb{N} \times \mathbb{Z}}=\left(a_{(i, j+N+t+L+1)}\right)_{(i, j) \in \mathbb{N} \times \mathbb{Z}} ;
$$

hence, for $\left(b_{(i, j)}\right)_{(i, j) \in \mathbb{N} \times \mathbb{Z}}:=\sigma_{\tau}^{N+t+L+1}\left(a_{(i, j)}\right)_{(i, j) \in \mathbb{N} \times \mathbb{Z}}$, we have

$$
b_{(p, q)}=a_{(p, q+N+t+L+1)}=d_{(p, q)} .
$$

So we have $\sigma_{\tau}^{N+t+L+1}\left(\left(a_{(i, j)}\right)_{(i, j) \in \mathbb{N} \times \mathbb{Z}}\right) \in V$. Thus, $\overline{\left\{\sigma_{\tau}^{n}\left(\left(a_{(i, j)}\right)_{(i, j) \in \mathbb{N} \times \mathbb{Z}}\right): n \geq 0\right\}}=\mathbb{N}^{\mathbb{N} \times \mathbb{Z}}$, and $\left(\mathbb{N}^{\mathbb{N} \times \mathbb{Z}}, \sigma_{\tau}\right)$ is point transitive.

Definition 9. The dynamical systems $(Z, f)$ and $(W, g)$ are topologically semiconjugate, if there exists an onto and continuous map, $\mu:(Z, f) \rightarrow(W, g)$ with $\mu \circ f=g \circ \mu$. Such a map $\mu:(Z, f) \rightarrow(W, g)$ is called a topological semiconjugacy. We call $(W, g)$ a factor of $(Z, f)$ if there exists a topological semiconjugacy $\mu:(Z, f) \rightarrow(W, g)$.
Lemma 10. In the dynamical systems $(Z, f)$ and $(W, g)$, if $\mu$ : $(Z, f) \rightarrow(W, g)$ is a topological semiconjugacy and $z_{0} \in Z$ is a transitive point of $(Z, f)$, then $\mu\left(z_{0}\right)$ is a transitive point of $(W, g)$.

In particular, the factor of a point transitive dynamical system is point transitive too.

Proof. Suppose $\mu:(Z, f) \rightarrow(W, g)$ is a topological semiconjugacy and $z_{0} \in Z$ is a point transitive of $(Z, f)$; we have

$$
W=\mu(Z)=\mu\left(\overline{O_{f}\left(z_{0}\right)}\right) \subseteq \overline{\mu\left(O_{f}\left(z_{0}\right)\right)}=\overline{O_{g}\left(\mu\left(z_{0}\right)\right)} \subseteq W
$$

therefore, $\overline{O_{g}\left(\mu\left(z_{0}\right)\right)}=W, \mu\left(z_{0}\right)$ is a transitive point of $(W, g)$, and $(W, g)$ is point transitive.

Lemma 11. For $\tau: \mathbb{N} \times \mathbb{Z} \rightarrow \mathbb{N} \times \mathbb{Z}$ with $\tau(n, m)=(n, m+$ 1) and countable $X=\left\{\theta_{1}, \theta_{2}, \ldots\right\}$, consider $\left(a_{(i, j)}\right)_{(i, j) \in \mathbb{N} \times \mathbb{Z}}$ as in Lemma 8; then, $\left(\theta_{a_{(n, m)}}\right)_{(n, m) \in \mathbb{N} \times \mathbb{Z}}$ is a transitive point of $\left(X^{\mathbb{N} \times \mathbb{Z}}, \sigma_{\tau}\right)$; in particular, $\left(X^{\mathbb{N} \times \mathbb{Z}}, \sigma_{\tau}\right)$ is point transitive.

Proof. We use the same notation for $\sigma_{\tau}: X^{\mathbb{N} \times \mathbb{Z}} \rightarrow X^{\mathbb{N} \times \mathbb{Z}}$ and $\sigma_{\tau}: \mathbb{N}^{\mathbb{N} \times \mathbb{Z}} \rightarrow \mathbb{N}^{\mathbb{N} \times \mathbb{Z}}$.

The map $g:\left(\mathbb{N}^{\mathbb{N} \times \mathbb{Z}}, \sigma_{\tau}\right) \rightarrow\left(X^{\mathbb{N} \times \mathbb{Z}}, \sigma_{\tau}\right)$ with

$$
g\left(\left(x_{(n, m)}\right)_{(n, m) \in \mathbb{N} \times \mathbb{Z}}\right)=\left(\theta_{x_{(n, m)}}\right)_{(n, m) \in \mathbb{N} \times \mathbb{Z}}
$$

is a semiconjugacy. By Lemma 8 and Definition 9 we have the desired result.

3.2. Main Construction. Now we are ready to have our main construction; suppose $\Gamma$ is infinite countable, $X=\left\{\theta_{1}, \theta_{2}, \ldots\right\}$ is countable, and $\left(X^{\Gamma}, \sigma_{\varphi}\right)$ is point transitive; hence, $\left(X^{\Gamma}, \sigma_{\varphi}\right)$ is topologically transitive and, by [2, Theorem 2.11], $\varphi: \Gamma \rightarrow \Gamma$ is one to one without any periodic point.

Using the same notations as in Theorem 3 and Definition 2 for $D \in \Lambda\left(=\Gamma / \sim_{\varphi}\right)$, we have $D=\alpha_{D} / \sim_{\varphi}=\bigcup\left\{\varphi^{n}\left(\alpha_{D}\right): n \in \mathbb{Z}\right\}$ and $D$ is infinite countable. Since $\Gamma$ is countable, $\Lambda$ is countable too and there exists a one to one map $\vartheta: \Lambda \rightarrow \mathbb{N}$.

Now define $\zeta: \Gamma \rightarrow \mathbb{N} \times \mathbb{Z}$ with $\zeta\left(\varphi^{n}\left(\alpha_{D}\right)\right)=(\vartheta(D), n)$ for all $D \in \Lambda$ and $n \in \mathbb{Z}$. Regarding $\zeta: \Gamma \rightarrow \mathbb{N} \times \mathbb{Z}$, we have the following facts:

(i) $\zeta: \Gamma \rightarrow \mathbb{N} \times \mathbb{Z}$ is well defined, since for every $\gamma \in \Gamma$ there exist unique $D \in \Lambda$ with $\gamma \in D$ and unique $n \in \mathbb{Z}$ with $\gamma=\varphi^{n}\left(\alpha_{D}\right)$;

(ii) $\zeta: \Gamma \rightarrow \mathbb{N} \times \mathbb{Z}$ is one to one, since $\vartheta$ is one to one.

Now consider $\left(X^{\mathbb{N} \times \mathbb{Z}}, \sigma_{\tau}\right)$ as in Lemma 11. For $\gamma \in \Gamma$, choose $n \in \mathbb{Z}$ and $D \in \Lambda$ with $\gamma=\varphi^{n}\left(\alpha_{D}\right)$; therefore,

$$
\begin{aligned}
\zeta(\varphi(\gamma)) & =\zeta\left(\varphi^{n+1}\left(\alpha_{D}\right)\right)=(\vartheta(D), n+1) \\
& =\tau(\vartheta(D), n)=\tau(\zeta(\gamma)) .
\end{aligned}
$$


Consider $v:\left(X^{\mathbb{N} \times \mathbb{Z}}, \sigma_{\tau}\right) \rightarrow\left(X^{\Gamma}, \sigma_{\varphi}\right)$ defined by $v\left(\left(x_{(n, m)}\right)_{(n, m) \in \mathbb{N} \times \mathbb{Z}}\right)=\left(x_{\zeta(\gamma)}\right)_{\gamma \in \Gamma}$; for $\left(x_{(n, m)}\right)_{(n, m) \in \mathbb{N} \times \mathbb{Z}}$, let $\left(y_{(n, m)}\right)_{(n, m) \in \mathbb{N} \times \mathbb{Z}}=\left(x_{(n, m+1)}\right)_{(n, m) \in \mathbb{N} \times \mathbb{Z}}$; we have

$$
\begin{aligned}
& v \sigma_{\tau}\left(\left(x_{(n, m)}\right)_{(n, m) \in \mathbb{N} \times \mathbb{Z}}\right) \\
& \quad=v\left(\left(x_{\tau(n, m)}\right)_{(n, m) \in \mathbb{N} \times \mathbb{Z}}\right)=v\left(\left(x_{(n, m+1)}\right)_{(n, m) \in \mathbb{N} \times \mathbb{Z}}\right) \\
& \quad=v\left(\left(y_{(n, m)}\right)_{(n, m) \in \mathbb{N} \times \mathbb{Z}}\right)=\left(y_{\zeta(\gamma)}\right)_{\gamma \in \Gamma} \\
& =\left(x_{\tau \zeta(\gamma)}\right)_{\gamma \in \Gamma}=\left(x_{\zeta \varphi(\gamma)}\right)_{\gamma \in \Gamma} \\
& =\sigma_{\varphi}\left(\left(x_{\zeta(\gamma)}\right)_{\gamma \in \Gamma}\right)=\sigma_{\varphi}\left(v\left(\left(x_{\alpha}\right)_{\alpha \in \Gamma}\right)\right) .
\end{aligned}
$$

Consider $\left(y_{\gamma}\right)_{\gamma \in \Gamma} \in X^{\Gamma}$ and $p \in X$; let

$$
x_{(m, n)}= \begin{cases}y_{\gamma} & (m, n)=\zeta(\gamma), \\ p & \text { otherwise }\end{cases}
$$

(This definition is well defined, since $\zeta: \Gamma \rightarrow \mathbb{N} \times \mathbb{Z}$ is one to one.)

Then, we have $v\left(\left(x_{(m, n)}\right)_{(m, n) \in \mathbb{N} \times \mathbb{Z}}\right)=\left(y_{\gamma}\right)_{\gamma \in \Gamma}$; thus, $v$ : $\left(X^{\mathbb{N} \times \mathbb{Z}}, \sigma_{\tau}\right) \rightarrow\left(X^{\Gamma}, \sigma_{\varphi}\right)$ is a semiconjugacy; hence, by Lemma 11 and Definition 9 and using the same notations, $\left(X^{\Gamma}, \sigma_{\varphi}\right)$ is point transitive, and

$$
v\left(\left(\theta_{a_{(n, m)}}\right)_{(n, m) \in \mathbb{N} \times \mathbb{Z}}\right)=\left(\theta_{a_{\zeta(\gamma)}}\right)_{\gamma \in \Gamma}
$$

is a transitive point of $\left(X^{\mathrm{\Gamma}}, \sigma_{\varphi}\right)$.
We recall that the dynamical system $(Z, h)$ with metric space $Z$ is called Devaney chaotic if $h: Z \rightarrow Z$ is sensitive dependence to initial conditions, the collection of periodic points of $h$ is dense in $Z$, and $(Z, h)$ is topological transitive [7]. Now note the fact that, for metric space $W$ with at least two elements, $W^{\Gamma}$ is metrizable if and only if $\Gamma$ is countable [8].

Remark 12. It has been proved in [2, Theorem 2.13] that for infinite countable $\Gamma$ the generalized shift dynamical system $\left(X^{\Gamma}, \sigma_{\varphi}\right)$ is Devaney chaotic, if and only if it is topological transitive.

Using Remark 12, Theorem 1, and the above construction, we have the following corollary, which shows an interaction between Devaney chaos and point transitivity in $\left(X^{\Gamma}, \sigma_{\varphi}\right)$.

Corollary 13. For infinite countable $\Gamma$ in the dynamical system $\left(X^{\Gamma}, \sigma_{\varphi}\right)$, the following statements are equivalent:

(i) the system $\left(X^{\Gamma}, \sigma_{\varphi}\right)$ is point transitive,

(ii) the set $X$ is countable and $\varphi: \Gamma \rightarrow \Gamma$ is one to one without any periodic point,

(iii) the set $X$ is countable and the system $\left(X^{\Gamma}, \sigma_{\varphi}\right)$ is Devaney chaotic.

Example 14. Let us return to our well known one-sided shift in the Introduction, that is, $\left(\{1, \ldots, k\}^{\mathbb{N}}, \sigma_{\kappa}\right)$ for $\kappa: \mathbb{N} \rightarrow \mathbb{N}$ with $\kappa(n)=n+1(n \in \mathbb{N})$, where $k \geq 2$. Since $\kappa$ is one to one without any periodic point, $\left(\{1, \ldots, k\}^{\mathbb{N}}, \sigma_{\kappa}\right)$ is point transitive by Corollary 13 (note that $\sigma_{\kappa}=\sigma$ in the Introduction). However, it is easy to see that

$$
(\underbrace{1,2}_{\text {all possible pairs in }\{1,2\}}, \underbrace{1,1,1,2,2,1,2,2}_{\text {all possible triples in }\{1,2\}}, 1,1,1,1,2,1,2,1,1,2,2,2,1,1,2,1,2,2,2,1,2,2,2, \ldots)
$$

is a transitive point of $\left(\{1,2\}^{\mathbb{N}}, \sigma_{\kappa}\right)$. However, using our method of construction and notations, for $\varphi=\kappa$ we have $\Lambda=\{\mathbb{N}\}$; thus, $D=\mathbb{N}$ is the unique element of $\Lambda$; we make the following choices (for fix $m$ ):

(a) $\alpha_{D}=1$,

(b) $\theta_{i}=i(i \geq 1)$,

(c) $\vartheta: \Lambda \rightarrow \mathbb{N}$, with $\vartheta(D)=m$.

$\zeta: \mathbb{N} \rightarrow \mathbb{N} \times \mathbb{Z}$ is $\zeta(n)=(m, n)$, so $\left(\theta_{a_{\zeta(n)}}\right)_{n \in \mathbb{N}}=\left(\theta_{a_{(m, n)}}\right)_{n \in \mathbb{N}}$ is a transitive point of $\left(\{1, \ldots, k\}^{\mathbb{N}}, \sigma_{\kappa}\right)$; however, we may find different transitive points by choosing another $m, \alpha_{D}$, or $\theta_{i} \mathrm{~s}$ in (a), (b), and (c).

\section{Conflict of Interests}

The authors declare that there is no conflict of interests regarding the publication of this paper.

\section{References}

[1] F. Ayatollah Zadeh Shirazi, N. Karami Kabir, and F. Heydari Ardi, "A note on shift theory," Mathematica Pannonica, vol. 19, no. 2, pp. 187-195, 2008.

[2] F. Ayatollah Zadeh Shirazi, J. Nazarian Sarkooh, and B. Taherkhani, "On Devaney chaotic generalized shift dynamical systems," Studia Scientiarum Mathematicarum Hungarica, vol. 50, no. 4, pp. 509-522, 2013.

[3] N. Değirmenci and Ş. Koçak, "Existence of a dense orbit and topological transitivity: when are they equivalent?" Acta Mathematica Hungarica, vol. 99, no. 3, pp. 185-187, 2003.

[4] S. Kolyada and L. Snoha, "Topological transitivity," Scholarpedia, vol. 4, no. 2, p. 5802, 2009.

[5] J.-H. Mai and W.-H. Sun, "Transitivities of maps of general topological spaces," Topology and Its Applications, vol. 157, no. 5, pp. 946-953, 2010.

[6] S. Silverman, "On maps with dense orbits and the definition of chaos," The Rocky Mountain Journal of Mathematics, vol. 22, no. 1, pp. 353-375, 1992. 
[7] R. L. Devaney, An Introduction to Chaotic Dynamical Systems, Addison-Wesley, 1989.

[8] J. R. Munkres, Topology: A First Course, Prentice-Hall, Englewood Cliffs, NJ, USA, 1974. 


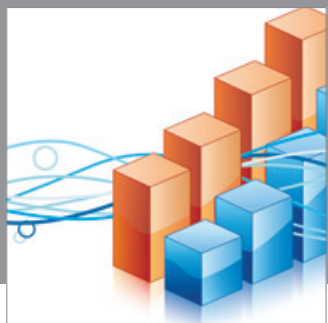

Advances in

Operations Research

mansans

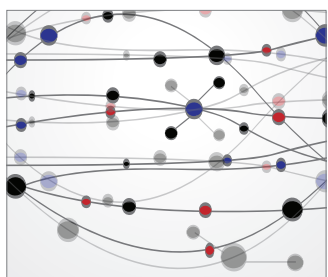

The Scientific World Journal
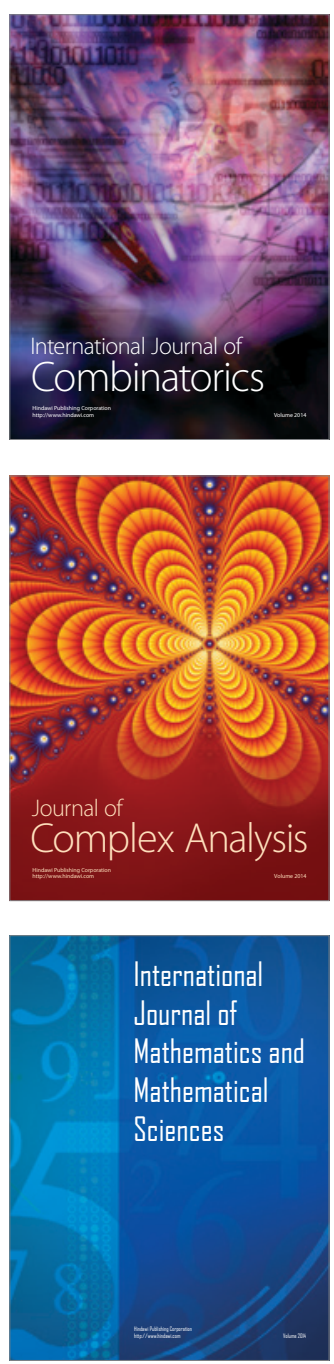
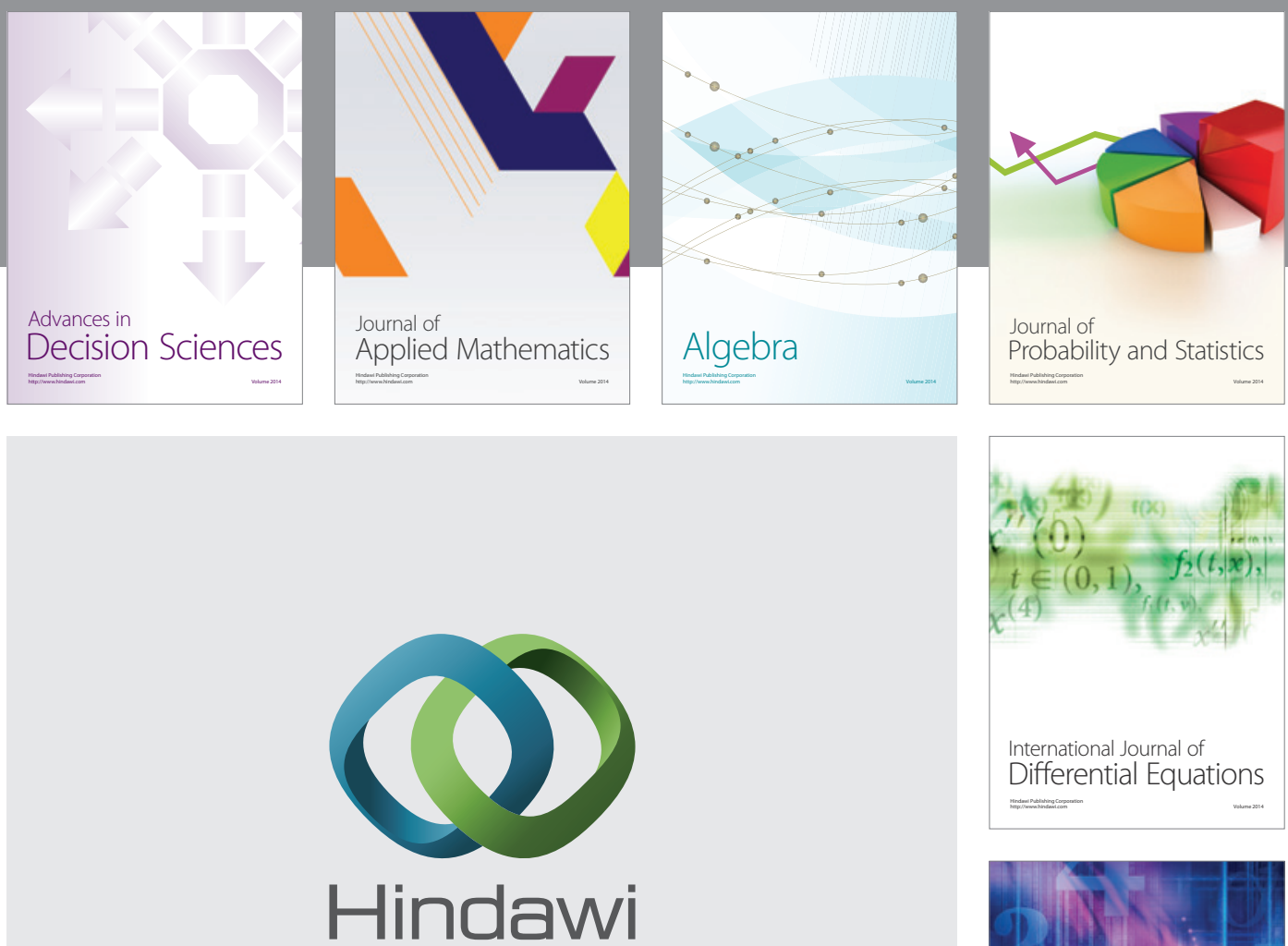

Submit your manuscripts at http://www.hindawi.com
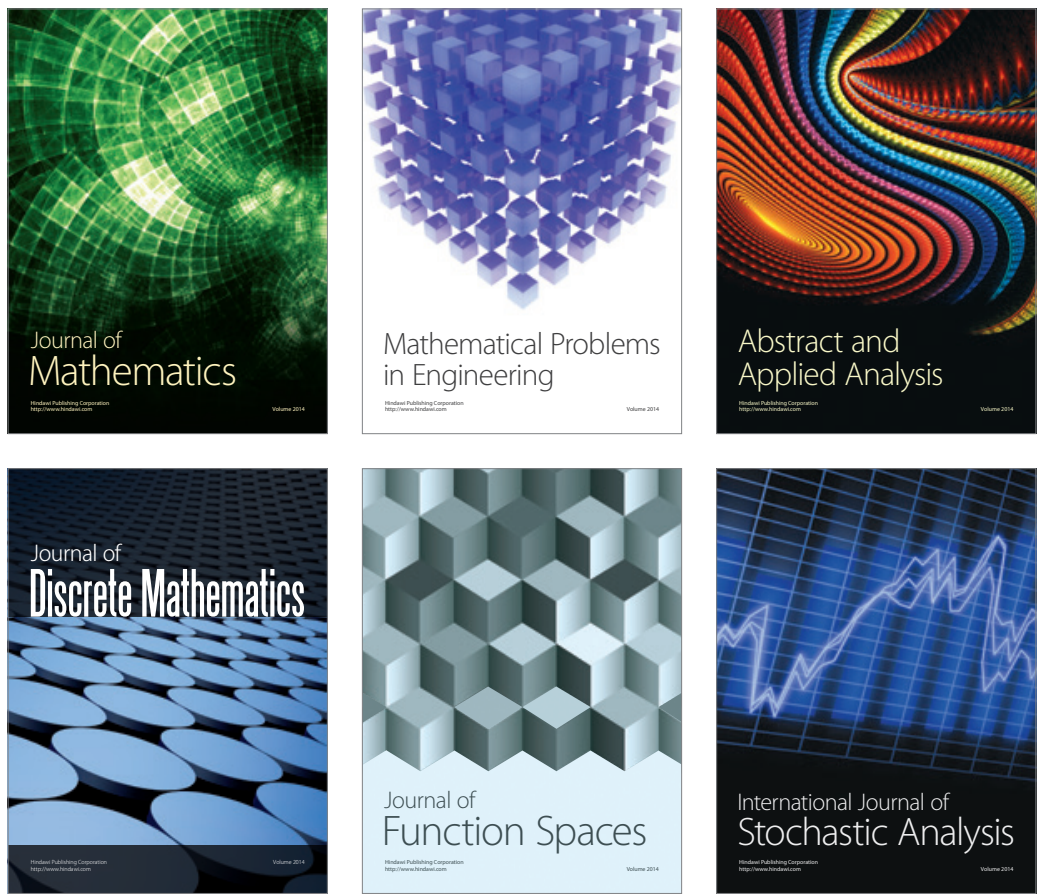

Journal of

Function Spaces

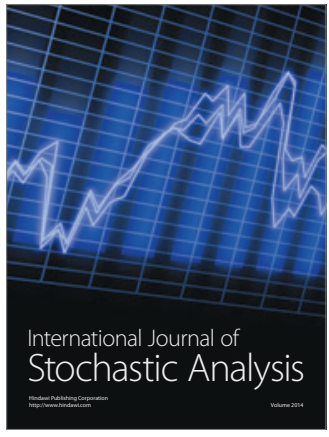

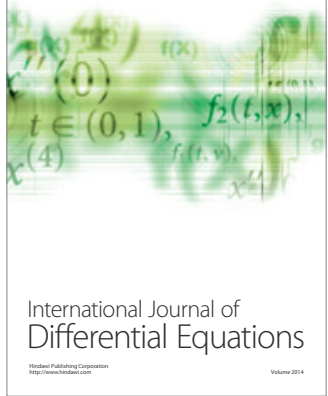
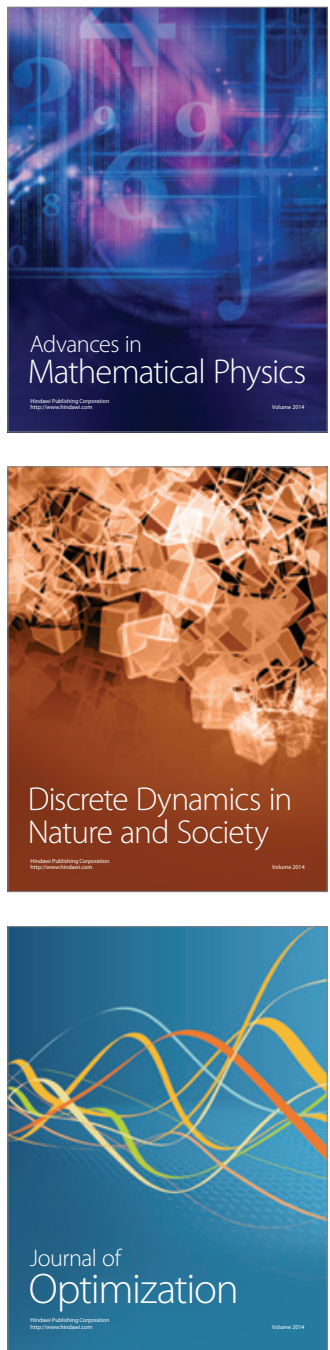